\title{
Diagnostic Value of C - Reactive Protein and Hematological Markers in Neonatal Sepsis
}

\author{
D. Jeyaganguli", G. Velvizhi, G. Sucilathangam and C. Revathy \\ Department of Microbiology, Tirunelveli Medical College, Tirunelveli - 627 011, \\ Tamil Nadu, India \\ *Corresponding author
}

\begin{tabular}{|l|}
\hline K e y w o r d s \\
Neonatal sepsis, C - \\
reactive protein \\
(CRP), Blood culture, \\
$\begin{array}{l}\text { Hematological } \\
\text { parameters }\end{array}$ \\
\hline Article Info \\
\hline $\begin{array}{l}\text { Accepted: } \\
\text { 07 February } 2018 \\
\text { Available Online: } \\
\text { 10 March 2018 }\end{array}$ \\
\hline
\end{tabular}

A B S T R A C T

Sepsis is a major problem in neonates. Clinical criteria alone could not establish the diagnosis of neonatal sepsis. It is extremely important to make an early diagnosis of neonatal sepsis for the prompt institution of anti-microbial therapy, which improves outcomes. The aim of this study is to determine the diagnostic performance of $\mathrm{C}$ - reactive protein (CRP) as early diagnostic marker in detection of neonatal sepsis in intensive neonatal care unit in comparison to that of blood culture and Hematological parameters like micro ESR and Total WBC count. This prospective study was conducted on neonates admitted to neonatal intensive care unit (NICU) at Tirunelveli Medical College Hospital, Tirunelveli, Tamil Nadu. Blood specimens $(n=60)$ were obtained from each neonate prior to commencement of antibiotics for sepsis work up including Hematological parameters like micro ESR, Total leukocyte count, absolute Neutrophil count (ANC), immature neutrophils to Total Neutrophil count ratio (I/T ratio). Blood culture was carried out by conventional method. Serum CRP level was measured by latex agglutination method. As per present study the sensitivity of CRP in predicting sepsis was $73.1 \%$, its specificity was $60 \%$, its positive predictive value was $57.6 \%$ and its negative predictive value was $77 \%$. $\mathrm{P}$ value of the test applied for statistical significance is less than 0.0001 which is statistically significant for CRP. Results of the current study indicate that despite the continuing emergence of newer markers of sepsis, CRP still plays a central role in the diagnosis of neonatal sepsis.

\section{Introduction}

Neonatal sepsis is defined as an invasive bacterial infection which occurs in the first 4 weeks of life. The incidence of neonatal sepsis varies from 11-24.5/1000 live births in India (Jaswal et al., 2003). The clinical manifestation of sepsis in newborn infants is usually non-specific. Neonatal sepsis is classified into early onset neonatal sepsis
(EONS) and late onset neonatal sepsis (LONS) according to time of onset of signs and symptoms. Early onset neonatal sepsis is defined as the onset of signs and symptoms within the first 72 hours of life. In late onset neonatal sepsis (LONS) clinical signs and symptoms occurs after 72 hours of age. Because of the high morbidity and mortality which is associated with neonatal sepsis (Bizzarro et al., 2005; Stoll et al., 2004), 
antibiotic therapy is commenced soon after the onset of the symptoms before the diagnosis is confirmed by blood culture.

Initial diagnosis of neonatal sepsis based on clinical signs and symptoms which are nonspecific as other non-infective condition like aspiration, asphyxia and metabolic disorders may also present with similar signs mimicking sepsis. The problem of symptom wise false positivity in diagnosing sepsis resulting in unwarranted initiation of empirical antibiotic therapy may lead to development of drug resistance, prolonged hospital stay, increased treatment cost and the separation of the neonates from their mothers.

The gold standard method for diagnosis of neonatal sepsis is isolation of microorganism from blood. It is time consuming procedure usually takes more than three days for complete result and also requires well equipped laboratory and trained personnel for better results. Hence alternative fast diagnostic test of serological markers enabling earlier detection of neonatal sepsis might be beneficial.

In addition to the blood culture, other tests that are usually used for the diagnosis of neonatal sepsis include estimations of the white-blood cell count (WBC), the absolute neutrophil count (ANC), micro ESR and the I/T ratio. Unfortunately, these tests do not have a high sensitivity and specificity in diagnosing neonatal sepsis.

Being exclusively produced in liver, CRP is an acute phase reactant whose level increases within 6 hours of acute inflammation, parallels the activity of inflammatory process, and then decreases faster than any other acute phase reactant. These characteristics make CRP very useful in diagnosing neonatal sepsis and monitoring response to antibiotics (Jaswal et al., 2003). CRP value is reliable in the first 24-
48 hours after the onset of infection. After obtaining a normal CRP level, the probability of sepsis is 10 times less likely. Similarly, for two successive normal CRP levels, the probability of sepsis becomes 30 times less likely (Bhandari, 2014).

\section{Materials and Methods}

This prospective study was conducted on neonates who were admitted to the neonatal intensive care unit (NICU) at Tirunelveli Medical College Hospital, Tirunelveli, Tamil Nadu over a period of 8 months. Informed consent was obtained from the parents. The inclusion criteria were infants who were admitted to this NICU with signs which is suggestive of sepsis, or those who developed signs of sepsis while they were in the ward. The exclusion criteria were infants who were on antibiotics or those who developed the signs of sepsis within 72 hours of discontinuation of the antibiotics and those who had birth asphyxia, aspiration syndromes, laboratory findings which were suggestive of the inborn errors of metabolism and congenital anomalies.

Blood was obtained from each neonate prior to the commencement of the antibiotics for the sepsis work up, which included hematological parameters like the microESR, total laeukocyte count, the absolute neutrophil count (ANC), the immature neutrophils to total neutrophil count ratio (I/T ratio), blood culture and C-reactive protein (CRP) estimation. Detection of serum level of CRP was done by latex agglutination test with the help CRP test kit of Agappe Diagnostics, India.

\section{Results and Discussion}

Based on the clinical findings and the laboratory data, sixty neonates who were eligible for the study were classified into two 
groups viz., probable sepsis (34) and proven sepsis (26 neonates). The results revealed that most of the neonates belong to EOS group. Total $59 \%$ of the cases were seen EOS and $41 \%$ cases were seen in LOS group. Gender distribution reveals that males constitute $58 \%$ are females constitute $42 \%$ with significant ratio of 1.3:1.

Out of the blood samples collected from 60 participants, 26 cases were found to be positive for blood culture. The organisms isolated were Klebsiella pneumoniae in 9 neonates (35\%), Escherichia coli in 6 neonates (23\%), Pseudomonas aeruginosa in 4 neonates (14\%), Klebsiella oxytoca in 3 neonates (12\%), and Staphylococcus aureus in 4 neonates (15\%). Klebsiella spp was found to be the most common organism in both early and late onset sepsis (Figure 1).

The hematological parameters in the sepsis groups were compared and presented in Table 1. The total WBC count was normal in 16 out of 26 cultures in the proven sepsis cases. An elevated micro ESR level was observed in seven proven sepsis neonate. An abnormal I/T ratio were observed in 6 proven sepsis cases.

In the present study $73 \%$ of the neonates with proven sepsis had raised CRP levels of more than $6 \mathrm{mg} / \mathrm{lit}$. About $44.1 \%$ of the neonates with suspected sepsis had raised CRP levels (Table 2).

The sensitivity of CRP in predicting sepsis was $73.1 \%$, its specificity was $60 \%$, its positive predictive value was $57.6 \%$ and its negative predictive value was $77 \%$, which is high compared to the sensitivity and specificity of other hematological parameters (micro ESR, I/T ratio and abnormal WBC count) (Table 3 ).

The neonatal sepsis is a leading cause of mortality and morbidity in neonates. Early diagnosis of neonatal sepsis helps the clinician in instituting antibiotic therapy at the earliest, thereby reducing the mortality rates in the neonates. The positive blood culture is the only definitive method of confirming a case of sepsis. Culture and sensitivity tests require a minimum period of 48 hours which is a precious time in making decisions in the treatment of sepsis in newborns. The readily achievable complete blood count and the leukocyte differential assays have a relatively poor specificity for diagnosing sepsis.

Therefore, the need persists for improved diagnostic indicators of neonatal sepsis.

Table.1 Relation between hematological parameters with sepsis groups

\begin{tabular}{|l|c|c|}
\hline \multicolumn{1}{|c|}{ Sepsis group } & Probable sepsis $(\mathbf{n = 3 4 )}$ & Proven sepsis $(\mathbf{n = 2 6})$ \\
\hline Test & & 7 \\
\hline Elevated micro ESR & 5 & 6 \\
\hline I:T Ratio & 4 & 10 \\
\hline Abnormal WBC count & 8 & \\
\hline
\end{tabular}

Table.2 Relation between CRP with sepsis groups

\begin{tabular}{|l|c|c|}
\hline $\begin{array}{l}\text { Sepsis group } \\
\text { CRP }\end{array}$ & Probable sepsis $(\mathbf{n = 3 4})$ & Proven sepsis $(\mathbf{n = 2 6 )}$ \\
\hline Positive & 14 & 19 \\
\hline Negative & 19 & 7 \\
\hline
\end{tabular}


Table.3 Comparison of CRP, haematological sepsis score and blood culture

\begin{tabular}{|l|c|c|}
\hline Parameter & No of Positive Out of $\mathbf{6 0}$ & No of positive (Out of 26) \\
\hline CRP & 34 & 19 \\
\hline Elevated Micro ESR & 12 & 7 \\
\hline I:T Ratio & 10 & 6 \\
\hline Abnormal WBC cout & 18 & 10 \\
\hline
\end{tabular}

Fig.1 Organism in blood culture

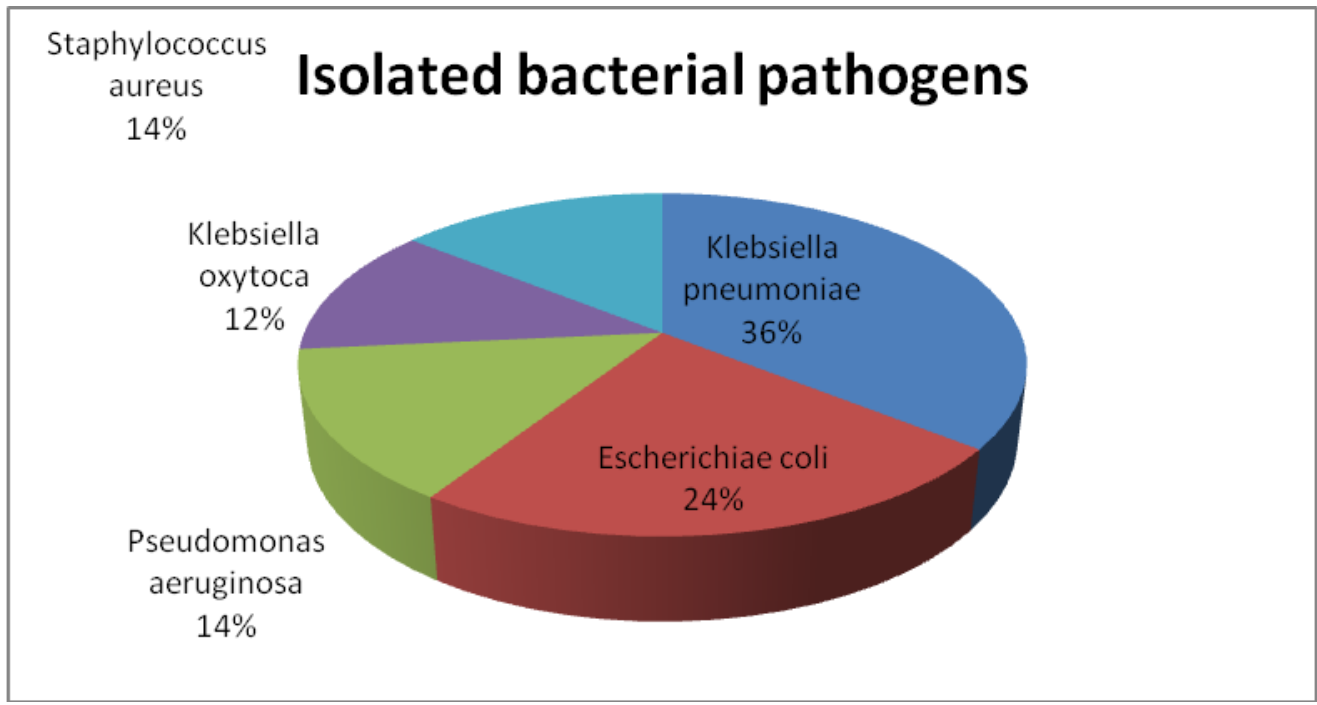

There is no single reliable test for the early definite diagnosis of neonatal sepsis, and therefore, there is a continuing search for a new infection marker. The $\mathrm{C}$ - reactive protein has been the most analyzed parameter for the detection of bacterial infections for years (Monneret et al., 2008; Cheisa et al., 1998).

The current study shows that among 60 suspected sepsis cases, blood culture was positive in 26 cases $(43.3 \%)$. Shrestha et al., in Nepal medical college, Kathmandu, during the period of July 2011 to January 2012 reported similar range results of $30.8 \%$ blood culture positive cases (Shrestha et al., 2013). In contrast low positivity ofblood culture had been reported in studies by Subhranshu SekaKar et al., in Bhubaneswar during the study period (2007-2010) which revealed $16.2 \%$ of blood culture positivity in 120 samples (Subhrashu et al., 2003). In the present study the total WBC count was abnormal in 10 out of $26(38.5 \%)$ proven sepsis cases. An elevated micro ESR level was observed in seven proven sepsis neonate. An abnormal I/T ratio was observed in 6 proven sepsis cases. In a similar study by Sucila Thangam et al., the total WBC count was abnormal in only one out of 13 cases with proven sepsis. An abnormal $\mathrm{I} / \mathrm{T}$ ratio was observed only in two cases (Sucilathangam et al., 2012) Similar results was observed in study by Rodwell et al., (1988), Zipursky et al., (1976) and Basu et al., (1999).

In the current study the sensitivity of CRP in predicting sepsis was $73.1 \%$, its specificity was $60 \%$, its positive predictive value was $57.6 \%$ and its negative predictive value was $77 \%$, which is high compared to the 
sensitivity and specificity of other haematological parameters (micro ESR, I/T ratio and abnormal WBC count). Similar studies done by Benitz et al., (2010) showed that C-reactive protein had higher sensitivity and specificity of $92.9 \%$ and $85 \%$ (Benitz, 2010) Manisha Yadav et al., in their study said that C-reactive protein was best single marker with an overall sensitivity and specificity of $88 \%$ and $84 \%$ (Manisha et al.,)

The marked difference of result among studies evaluating C-reactive protein as useful marker can be explained by non-availability of universally acceptable definition of neonatal sepsis, difference in reference range values and environmental influence on the results in different setups. The test for estimation of CRP is easy to perform and results will be available in minutes. Furthermore, it can also be used effectively in neonates who had already used antibiotics.

CRP is one of the most widely available, most studied, and most used laboratory tests for neonatal bacterial infection and despite the continuing emergence of new markers of infection, it still plays a central role in the diagnosis of neonatal sepsis. Although blood culture is still a gold standard test in diagnosing sepsis, its main drawback is its delayed result, more chances of contamination, high cost and non-availability in most peripheral setups in our country. Our study suggests that CRP could be used as a preferred marker in evaluating a neonate for sepsis. CRP along with other laboratory tests and physical examination will be useful in identifying infants with a low probability of infection.

\section{Acknowledgement}

The authors are gratefully acknowledged The Dean, Tirunelveli Medical College Hospital, Tirunelveli, Tamil Nadu, The Staff of
Paediatrics and The Staff of Microbiology, Tirunelveli Medical College Hospital.

\section{References}

Basu S, Guruprasad, Narang A, Garewal G. The diagnosis of sepsis in high risk neonates by using a hematologic scoring system. Indian $\mathrm{J}$ Hematolo Blood Transfusion 1999; 17: 32-34.

Bhandari V. Effective biomarkers for diagnosis of neonatal sepsis. [5] J Pediatric Infect Dis Soc. 2014; 3: 23445

Bizzarro MJ, Raskind C, Baltimore RS, Gallagher PG. Seventy five years of neonatal sepsis at Yale: 1928-2003. Paediatrics 2005; 116: 595-602.

Cheisa, C., et al., Procalcitonin in pediatrics; overview and challenge. Infection 26 (1998); No 4: 236-240.

Jaswal RS, Kaushal RK, Goel A, Pathania K. Role of C-reactive [4] protein in deciding duration of antibiotic therapy in neonatal septicaemia. Indian Pediatr. 2003; 40: 800-03.

Jaswal RS, Kaushal RK, Goel A, Pathania K. Role of the C-reactive protein in deciding the duration of the antibiotic therapy in neonatal septicaemia. Indian Paediatrics 2003; 40: 800-83.

Manisha Y, Rosy L, Sachin D. Journal of Current Research, 7(7), 18280-18282

Monneret G, Labaunejm et al., 2008. Procalcitonin and CRP levels in neonatal infections. ActaPediatri; 1997 Feb, 86(2): 209 -212.

Rodwell RL, Leslie AL, Tudehope DL. Early diagnosis of neonatal sepsis by using a hematological scoring system. J Paediatr 1988; 112: 16166.

Shrestha R K, Rai S K and Mandhal P K. Bacteriological study of neo natal sepsis and antibiotic susceptibility pattern of isolates in Kathmandu, Nepal. Nepal Med Coll J 2013; 15(1): 71-73. 
Stoll BJ, Hansen NI, Adams-Chapman I, Fanaroff AA, Hintz SR, Vohr B, Higgins RD. Neurodevelopmental and growth impairment among extremely low-birth-weight infants with neonatal infections. JAMA 2004; 292:2357-65.

Subhrashu S, Rajani D, Samarendra M, Sitanshu S. The role of clinical signs in the diagnosis of late onset neonatal sepsis and formation of clinical score. Indian J clinPrac, 2003 Mar; Vol.23, No 23: 654-660.

Sucilathangam G., Amuthavalli K., Velvizhi

G., Ashihabegum M.A., Jeyamurugan

T., Palaniappan N. Early Diagnostic
Markers for Neonatal Sepsis: Comparing Procalcitonin (PCT) and C reactive protein (CRP). Journal of Clinical and Diagnostic Research. 2012 May (Suppl-2), Vol-6(4): 627-631.

William E. Benitz. Adjunct Laboratory Tests in the Diagnosis of Early-Onset Neonatal Sepsis. Clinics in Perinatology. 2010; 37(2):421-438.

Zipursky A, Palko J, Milner R, Akenzua GI. The hematology of the bacterial infections in premature infants. Paediatrics 1976, 57: 83953.

\section{How to cite this article:}

Jeyaganguli, D., G. Velvizhi, G. Sucilathangam and Revathy, C. 2018. Diagnostic Value of C Reactive Protein and Hematological Markers in Neonatal Sepsis. Int.J.Curr.Microbiol.App.Sci. 7(03): 722-727. doi: https://doi.org/10.20546/ijcmas.2018.703.084 